ESAIM: PROCEEDINGS, December 2013, Vol. 43, p. 180-194

S. Descombes, B. Dussoubs, S. Faure, L. Gouarin, V. Louvet, M. Massot, V. Miele, Editors

\title{
DIVERGENCE-FREE MHD SIMULATIONS WITH THE HERACLES CODE
}

\author{
J. Vides ${ }^{1}$, E. Audit ${ }^{2}$, H. Guillard ${ }^{3}$ Et B. NkongA ${ }^{4}$
}

Résumé. Au fil des ans, la simulation numérique des équations de la magnétohydrodynamique (MHD) a joué un rôle important dans la recherche en physique des plasmas. La nécessité de trouver des solutions physiques et stables à ces équations a conduit à l'élaboration de plusieurs schémas numériques, tous devant satisfaire et préserver la contrainte de divergence nulle pour le champ magnétique numérique. Dans cet article, nous tenterons de montrer l'importance de maintenir cette contrainte numériquement. En particulier, nous étudions la technique de nettoyage hyperbolique de la divergence appliquée aux équations de la MHD idéale discrétisées sur une grille colocalisée et nous la comparons à la technique du transport constraint qui utilise une grille décalée pour maintenir cette propriété. Les méthodes sont implémentées dans le code HERACLES et des tests numériques sont présentés. Il est ainsi possible de comparer directement la robustesse et la précision des méthodes.

\begin{abstract}
Numerical simulations of the magnetohydrodynamics (MHD) equations have played a significant role in plasma research over the years. The need of obtaining physical and stable solutions to these equations has led to the development of several schemes, all requiring to satisfy and preserve the divergence constraint of the magnetic field numerically. In this paper, we aim to show the importance of maintaining this constraint numerically. We investigate in particular the hyperbolic divergence cleaning technique applied to the ideal MHD equations on a collocated grid and compare it to the constrained transport technique that uses a staggered grid to maintain the property. The methods are implemented in the software HERACLES and several numerical tests are presented, where the robustness and accuracy of the different schemes can be directly compared.
\end{abstract}

\section{INTRODUCTION}

The governing equations of magnetohydrodynamics (MHD) are used to model electrically conducting fluid flows in the presence of magnetic fields. Given the ubiquity of these flows and the simplicity of the model, MHD has widespread application in both astrophysical and magnetically confined fusion plasmas. In the field of plasma physics, the MHD model allows to treat plasma as a single conducting fluid and describe different phenomena using macroscopic quantities and a corresponding system of conservation laws. Experimentally, these modeled phenomena are found to closely approximate aspects of real plasma behavior, such as MHD equilibria and stability, Alfvén waves, and field line freezing, among others.

Therefore, it is not surprising that in the last few decades, the desire of performing highly efficient MHD simulations has become increasingly important. In order to have robust, accurate and stable solutions, it is necessary to satisfy the solenoidal property of the magnetic field, which requires the magnetic field to vanish

${ }^{1}$ Inria, Maison de la Simulation, USR 3441, Gif-sur-Yvette, France. e-mail : jeaniffer-lissette.vides_higueros@inria.fr

2 CEA, Maison de la Simulation, USR 3441, Gif-sur-Yvette, France. e-mail : edouard.audit@cea.fr

${ }^{3}$ Université de Nice-Sophia Antipolis, UMR CNRS 7351 \& Inria Sophia Antipolis, France. e-mail : herve.guillard@inria.fr

${ }^{4}$ Université de Nice-Sophia Antipolis, UMR CNRS 7351 \& Inria Sophia Antipolis, France. e-mail : boniface.nkonga@unice.fr

(c) EDP Sciences, SMAI 2013 
everywhere at all times, while maintaining the conservation form of the fundamental physical laws. However, it is widely known that special care needs to be taken to satisfy and control this property on any numerical scheme, even if the magnetic field is initially divergence-free. Failure to do so may result in nonlinear numerical instabilities and discretization errors increasing over time, manifesting themselves as discrepancies in the simulations, e.g., incorrect jump conditions, wrong propagation speed of discontinuities, appearance of unphysical effects such as plasma transport orthogonal to the magnetic field and negative pressures and/or densities.

The conservation law formulation of the MHD equations allows the use of Godunov-type schemes for their solution, and as a consequence, several strategies that aim to maintain the divergence-free property in multidimensional Godunov-type codes have been developed over several years. In this paper, we focus on the divergence cleaning and constrained transport (CT) methods. The latter, originally introduced by Evans and Hawley [6], involves the use of a staggered magnetic field, with its components defined at the cell interfaces. It is well known that this method provides a natural expression for the induction equation in conservative form. Hence, the combination of the CT framework with the Godunov one is an attractive solution, see $[1,4,6,7]$, and this is the reason why it was the default and only technique used in the HERACLES code in order to perform MHD simulations. However, the staggered collocation of magnetic and electric field variables, makes the use of this method in unstructured grids rather laborious and costly.

One of our future goals is to design a high order finite volume approximation for hyperbolic conservation laws in curvilinear unstructured grids. Given that HERACLES is currently a structured mesh code, we believe that other methods that do not involve a staggered grid will be simpler to extend to unstructured grids. Thus, we are motivated by the need to explore an alternative method and implement it in the HERACLES code. Among the different existing techniques, we choose to investigate the hyperbolic cleaning method introduced by Dedner et al. [5]. The main advantage of using this method is that it is easy to implement, since it is completely based on the cell-centered discretizations favored in Godunov schemes, and thus allows highly accurate solutions with reduced computational effort. We then compare both methods using several tests that aim to put in evidence their advantages and disadvantages.

This paper is organized as follows. In the next section, we review the governing equations for ideal MHD flows, which treat plasma as a perfectly conducting fluid, and stress the importance of maintaining the divergence-free constraint at all times when performing numerical simulations. Some standard notation is introduced briefly in Section 2. The details of the hyperbolic divergence cleaning method and the constrained transport methods are presented in Sections 3 and 4, respectively. Several numerical tests are presented and discussed in Section 5. Finally, concluding remarks are given in Section 6.

\section{Governing EQuAtions And The Divergence-FreE CONDITION}

The ideal MHD equations are a set of nonlinear hyperbolic equations in conservation form, given by

$$
\begin{aligned}
\partial_{t} \rho+\nabla \cdot(\rho \mathbf{u}) & =0 \\
\partial_{t}(\rho \mathbf{u})+\nabla \cdot(\rho \mathbf{u} \otimes \mathbf{u})+\nabla\left(p+\frac{1}{2} \mathbf{B} \cdot \mathbf{B}\right)-\nabla \cdot(\mathbf{B} \otimes \mathbf{B}) & =0 \\
\partial_{t} \varepsilon+\nabla \cdot\left[\left(\varepsilon+p+\frac{1}{2} \mathbf{B} \cdot \mathbf{B}\right) \mathbf{u}-\mathbf{B}(\mathbf{u} \cdot \mathbf{B})\right] & =0 \\
\partial_{t} \mathbf{B}+\nabla \cdot(\mathbf{B} \otimes \mathbf{u}-\mathbf{u} \otimes \mathbf{B}) & =0
\end{aligned}
$$

where $\rho$ and $\mathbf{u}=\left(u_{x}, u_{y}, u_{z}\right)$ are the fluid density and velocity, respectively, and $\mathbf{B}=\left(B_{x}, B_{y}, B_{z}\right)$ is the magnetic field. Moreover, the magnetic field satisfies the constraint

$$
\nabla \cdot \mathbf{B}=0
$$


which will be discussed further below in Section 1.1. The total energy density $\varepsilon$ and the thermal pressure $p$ are related through the ideal gas law

$$
p=(\gamma-1)\left(\varepsilon-\frac{\rho}{2} \mathbf{u} \cdot \mathbf{u}-\frac{1}{2} \mathbf{B} \cdot \mathbf{B}\right),
$$

which completes the set of equations. Unless stated otherwise, we will assume throughout the paper, that the specific heat capacity ratio $\gamma$ is $5 / 3$. The evolution equation for the magnetic field (4) is conveniently written in divergence form and it comes from Faraday's law:

$$
\partial_{t} \mathbf{B}+\nabla \times \mathbf{E}=0,
$$

with the electric field $\mathbf{E}$ given by the ideal Ohm's law

$$
\mathbf{E}=-\mathbf{u} \times \mathbf{B} .
$$

\subsection{Divergence constraint}

The constraint $\nabla \cdot \mathbf{B}=0$ is not necessary in the time evolution in the sense that if the magnetic field is assumed at the initial time step to be divergence-free, then an exact solution to the MHD equations will satisfy this condition for all times $t>0$. For smooth solutions, this is guaranteed by the evolution equation (4), since taking the divergence of the equivalent equation (6) and recalling that $\nabla \cdot(\nabla \times) \equiv 0$, gives

$$
\partial_{t}(\nabla \cdot \mathbf{B})=0 .
$$

As a result, from an analytical point of view, we sometimes find in the literature that equation (5) is regarded as an involution rather than a constraint, as in $[2,8]$. Ideally, when performing numerical simulations, we would expect this particular equation to remain zero at all times. This is the case in one dimension, where the constraint becomes $\partial_{x} B_{x}=0$ and the evolution equation for $B_{x}$ in (4), decoupled from the other equations, is reduced to $\partial_{t} B_{x}=0$. Hence, an initial $\partial_{x} B_{x}(\cdot, 0)=0$ leads to $\partial_{x} B_{x}(\cdot, t)=0$ for all times $t>0$. However, the matter is more complicated for multidimensional MHD flows. As detailed by the work of Brackbill and Barnes [3], numerical discretization errors have an impact on its time evolution in the following way:

$$
\partial_{t}(\nabla \cdot \mathbf{B})=0+\mathcal{O}\left((\Delta x)^{m},(\Delta t)^{n}\right),
$$

where $\Delta x$ and $\Delta t$ are respectively the space and time discretization steps and $m, n \geq 1$. In the same paper, Brackbill and Barnes show the importance of choosing an appropriate discretization of (5) in order to avoid the emergence of unwanted and unphysical effects in the MHD system. Basically, if $\nabla \cdot \mathbf{B} \neq 0$, the magnetic force F defined by

$$
\mathbf{F}=\nabla \cdot(\mathbf{B} \otimes \mathbf{B})-\frac{1}{2} \nabla(\mathbf{B} \cdot \mathbf{B}),
$$

will not in general disappear in the direction of the magnetic field, i.e.,

$$
\mathbf{F} \cdot \mathbf{B}=(\nabla \cdot \mathbf{B})(\mathbf{B} \cdot \mathbf{B}) \neq 0 .
$$

Therefore, the behavior of the system may become unphysical due to an increase of spurious forcing, leading to instabilities. In general, the effects of not controlling the numerical errors arising from the discrete form of the divergence-free constraint have been well-documented in the literature, and interesting examples and conclusions can be found in $[1,3,5,14]$, as well as in Section 5 in this paper. For the moment, we only mention that, from a numerical point of view, $\nabla \cdot \mathbf{B}=0$ represents a constraint which cannot be safely ignored. 


\section{General Notation}

In this section, we introduce the notation as a standard for the numerical approximations of both the divergence cleaning and constrained transport techniques. We consider a uniform numerical grid in a threedimensional domain with Cartesian coordinates $(x, y, z)$. Henceforth, superscripts denote time levels and subscripts refer to spatial location. The cell with center at $\left(x_{i}, y_{j}, z_{k}\right)$ is denoted by the integer subscripts $(i, j, k)$ and the centers of the cell's interfaces are denoted by half integers. We consider a system of conservation laws of the form

$$
\partial_{t} \mathbf{U}+\nabla \cdot \mathcal{F}(\mathbf{U})=0
$$

with the flux $\mathcal{F}=(\mathbf{F}, \mathbf{G}, \mathbf{H})$. If we integrate this system in a grid cell $x_{i-1 / 2} \leq x \leq x_{i+1 / 2}, y_{j-1 / 2} \leq y \leq y_{j+1 / 2}$, $z_{k-1 / 2} \leq z \leq z_{k+1 / 2}$ and over a time step $t^{n} \leq t \leq t^{n+1}$, we obtain the following expression:

$$
\mathbf{U}_{i, j, k}^{n+1}=\mathbf{U}_{i, j, k}^{n}-\frac{\Delta t}{\Delta x}\left[\mathbf{F}_{i+1 / 2, j, k}^{n}-\mathbf{F}_{i-1 / 2, j, k}^{n}\right]-\frac{\Delta t}{\Delta y}\left[\mathbf{G}_{i, j+1 / 2, k}^{n}-\mathbf{G}_{i, j-1 / 2, k}^{n}\right]-\frac{\Delta t}{\Delta z}\left[\mathbf{H}_{i, j, k+1 / 2}^{n}-\mathbf{H}_{i, j, k-1 / 2}^{n}\right]
$$

where $\Delta x, \Delta y$ and $\Delta z$ are the mesh sizes in each direction and the time increment is given by $\Delta t$, such that $t^{n+1}=t^{n}+\Delta t$. In equation (9), both $\mathbf{U}_{i, j, k}^{n}$ and $\mathbf{U}_{i, j, k}^{n+1}$ are cell-averaged values of $\mathbf{U}$ at time $t^{n}$ and $t^{n+1}$, respectively, and the fluxes are obtained by a time-surface average. In a Godunov-type scheme, the fluxes in equation (9) are evaluated by solving a one-dimensional Riemann problem $\mathcal{R}$ in the normal direction $\hat{n}$ at each cell interface. Thus, in the $x$-direction, we have

$$
\mathbf{F}_{i+1 / 2, j, k}^{n}=\mathbf{F}\left(\mathcal{R}\left(0 ; \mathbf{U}_{i, j, k}, \mathbf{U}_{i+1, j, k}\right)\right) \quad \text { and } \quad \mathbf{F}_{i-1 / 2, j, k}^{n}=\mathbf{F}\left(\mathcal{R}\left(0 ; \mathbf{U}_{i-1, j, k}, \mathbf{U}_{i, j, k}\right)\right),
$$

where $\mathcal{R}\left(x / t ; \mathbf{U}_{i, j, k}, \mathbf{U}_{i+1, j, k}\right)$ is the approximate solution of the Riemann problem at $x_{i+1 / 2}$. Similar expressions can be found for the fluxes in the remaining directions.

\section{Hyperbolic DiVERGENCE CLEANING}

When all variables defined in the hyperbolic system (1)-(4) are defined in the same position, a cleaning technique is needed to enforce the constraint $\nabla \cdot \mathbf{B}=0$. The hyperbolic divergence cleaning method suggested by Dedner et al. [5] is based on coupling the divergence constraint (5) to the evolution equation for the magnetic field (4) by introducing a new scalar function or generalized Lagrangian multiplier (GLM) $\psi$. Then, both of the mentioned equations, are replaced by

$$
\begin{aligned}
\partial_{t} \mathbf{B}+\nabla \cdot(\mathbf{B} \otimes \mathbf{u}-\mathbf{u} \otimes \mathbf{B})+\nabla \psi & =0, \\
\mathcal{D}(\psi)+\nabla \cdot \mathbf{B} & =0,
\end{aligned}
$$

with $\mathcal{D}(\cdot)$ being a linear differential operator. Henceforth, the resulting system (1), (2), (3), (11), (12) is called the generalized Lagrange multiplier (GLM) formulation of the MHD equations, or simply, GLM-MHD. Dedner et al. analyzed different possibilities for $\mathcal{D}$ and found that a satisfactory approximation to the original system may be obtained by choosing a mixed hyperbolic/parabolic ansatz, which will be explained in detail in Section 3.1. Additionally, in order to obtain a good numerical approximation, it is necessary to choose adequate initial and boundary conditions for the unphysical variable $\psi$ (see Section 3.4). We keep the notation used by Dedner et al. with few minor changes.

\subsection{Linear differential operator $\mathcal{D}$}

Let us consider sufficiently smooth solutions. From equations (11) and (12), we can deduce that for any choice of $\mathcal{D}$, the divergence of the magnetic field and the scalar function $\psi$ satisfy the same equation

$$
\begin{array}{cll}
\partial_{t} \mathcal{D}(\nabla \cdot \mathbf{B}) & -\Delta(\nabla \cdot \mathbf{B}) & =0, \\
\partial_{t} \mathcal{D}(\psi) & -\Delta \psi & =0 .
\end{array}
$$




\subsubsection{Parabolic correction}

Defining the linear differential operator as

$$
\mathcal{D}(\psi)=\frac{1}{c_{p}^{2}} \psi
$$

with $c_{p} \in(0, \infty)$, and using it in (14) yields the heat equation $\partial_{t} \psi-c_{p}^{2} \Delta \psi=0$. Hence, this type of correction allows for the perturbations in the magnetic field to be dissipated and smoothed out, if appropriate boundary conditions are defined. However, the explicit approximation to the MHD equations using a parabolic correction presents certain difficulties due to the restrictions imposed on the parameter $c_{p}$ by stability conditions. Since we are only interested in explicit schemes, we study more suitable operators.

\subsubsection{Hyperbolic correction}

We obtain a hyperbolic correction by choosing

$$
\mathcal{D}(\psi)=\frac{1}{c_{h}^{2}} \partial_{t} \psi,
$$

with $c_{h} \in(0, \infty)$. Substituting (16) into (14) gives the wave equation $\partial_{t t}^{2} \psi-c_{h}^{2} \Delta \psi=0$. Thus, local divergence errors are transported to the boundary with finite speed $c_{h}$. Now, if we express equation (12) in terms of the hyperbolic correction, we obtain

$$
\partial_{t} \psi+c_{h}^{2}(\nabla \cdot \mathbf{B})=0
$$

which is an attractive result since the resulting GLM-MHD system is purely hyperbolic.

\subsubsection{Mixed correction}

Formally, this approach is nothing but the combination of the parabolic and hyperbolic corrections, with the linear differential operator defined by

$$
\mathcal{D}(\psi)=\frac{1}{c_{h}^{2}} \partial_{t} \psi+\frac{1}{c_{p}^{2}} \psi,
$$

where $c_{p}$ and $c_{h}$ are the parabolic and hyperbolic constants previously defined. Direct substitution of this correction into (14) leads to the telegraph equation $\partial_{t t}^{2} \psi+c_{h}^{2} / c_{p}^{2} \partial_{t} \psi=c_{h}^{2} \Delta \psi$, which implies that the errors associated to the divergence of the magnetic field are both transported with speed $c_{h}$ and damped with time and distance. Following the same approach used for the other corrections, from (14), we get

$$
\partial_{t} \psi+c_{h}^{2}(\nabla \cdot \mathbf{B})=-\frac{c_{h}^{2}}{c_{p}^{2}} \psi,
$$

where it is evident that the damping comes now from a source term.

\subsection{Eigensystem of the GLM-MHD equations}

The complete GLM-MHD system with the mixed correction (18) can be written in the following form:

$$
\partial_{t}\left(\begin{array}{c}
\rho \\
\rho \mathbf{u} \\
\mathbf{B} \\
\varepsilon \\
\psi
\end{array}\right)+\nabla \cdot\left(\begin{array}{c}
\rho \mathbf{u} \\
\rho \mathbf{u} \otimes \mathbf{u}+\left(p+\frac{1}{2} \mathbf{B} \cdot \mathbf{B}\right) \mathcal{I}-\mathbf{B} \otimes \mathbf{B} \\
\mathbf{B} \otimes \mathbf{u}-\mathbf{u} \otimes \mathbf{B}+\psi \mathcal{I} \\
\left(\varepsilon+p+\frac{1}{2} \mathbf{B} \cdot \mathbf{B}\right) \mathbf{u}-\mathbf{B}(\mathbf{u} \cdot \mathbf{B}) \\
c_{h}^{2} \mathbf{B}
\end{array}\right)=\left(\begin{array}{c}
0 \\
0 \\
0 \\
0 \\
-\frac{c_{h}^{2}}{c_{p}^{2}} \psi
\end{array}\right)
$$


where $\mathcal{I}$ is a $3 \times 3$ identity matrix. This system, with a source term only in the equation for the unphysical variable $\psi$, can be written in compact form as

$$
\partial_{t} \mathbf{V}+\nabla \cdot \mathcal{G}(\mathbf{V})=\mathcal{S}(\mathbf{V})
$$

with $\mathbf{V}=(\rho, \rho \mathbf{u}, \mathbf{B}, \varepsilon, \psi)^{T}$ and the flux function $\mathcal{G}=\left(\mathcal{G}_{x}, \mathcal{G}_{y}, \mathcal{G}_{z}\right)$. Note that, in the limiting case where $c_{p} \rightarrow \infty$, the mixed correction reduces to the hyperbolic one and $\mathcal{S}(\mathbf{V})=0$. Moreover, given the primitive variables $\mathbf{W}=\left(\rho, u_{x}, u_{y}, u_{z}, B_{x}, B_{y}, B_{z}, p, \psi\right)^{T}$, the homogeneous version of equation (21) may be rewritten in the quasilinear form

$$
\partial_{t} \mathbf{W}+\mathcal{A}(\mathbf{W}) \partial_{x} \mathbf{W}+\mathcal{B}(\mathbf{W}) \partial_{y} \mathbf{W}+\mathcal{C}(\mathbf{W}) \partial_{z} \mathbf{W}=0
$$

where, for example,

$$
\mathcal{A}(\mathbf{W})=\left(\begin{array}{ccccccccc}
u_{x} & \rho & 0 & 0 & 0 & 0 & 0 & 0 & 0 \\
0 & u_{x} & 0 & 0 & -\frac{B_{x}}{\rho} & \frac{B_{y}}{\rho} & \frac{B_{z}}{\rho} & \frac{1}{\rho} & 0 \\
0 & 0 & u_{x} & 0 & -\frac{B_{y}}{\rho} & -\frac{B_{x}}{\rho} & 0 & 0 & 0 \\
0 & 0 & 0 & u_{x} & -\frac{B_{z}}{\rho} & 0 & -\frac{B_{x}}{\rho} & 0 & 0 \\
0 & 0 & 0 & 0 & 0 & 0 & 0 & 0 & 1 \\
0 & B_{y} & -B_{x} & 0 & -u_{y} & u_{x} & 0 & 0 & 0 \\
0 & B_{z} & 0 & -B_{x} & -u_{z} & 0 & u_{x} & 0 & 0 \\
0 & \gamma p & 0 & 0 & (\gamma-1) \mathbf{u} \cdot \mathbf{B} & 0 & 0 & u_{x} & (1-\gamma) B_{x} \\
0 & 0 & 0 & 0 & c_{h}^{2} & 0 & 0 & 0 & 0
\end{array}\right) .
$$

In the matrix $\mathcal{A}$ defined above, we see that it is possible to decouple the equations for $B_{x}$ and $\psi$ from the remaining system and solve them independently. Thus, for a one-dimensional problem, we obtain the following decoupled system of equations:

$$
\partial_{t}\left(\begin{array}{c}
B_{x} \\
\psi
\end{array}\right)+\left(\begin{array}{cc}
0 & 1 \\
c_{h}^{2} & 0
\end{array}\right) \partial_{x}\left(\begin{array}{c}
B_{x} \\
\psi
\end{array}\right)=\left(\begin{array}{l}
0 \\
0
\end{array}\right) .
$$

Additionally, given $\mathbf{W}^{\prime}=\left(\rho, u_{x}, u_{y}, u_{z}, B_{y}, B_{z}, p\right)^{T}$, we define the matrix $\mathcal{A}^{\prime}\left(\mathbf{W}^{\prime}\right)$ by removing the fifth and ninth row and column from $\mathcal{A}(\mathbf{W})$. Considering $B_{x}$ as a constant parameter, we get the quasilinear system

$$
\partial_{t} \mathbf{W}^{\prime}+\mathcal{A}^{\prime}\left(\mathbf{W}^{\prime}\right) \partial_{x} \mathbf{W}^{\prime}=0
$$

It is well known that matrix $\mathcal{A}^{\prime}$ is diagonalizable and has seven eigenvalues corresponding to one entropy wave traveling with speed $\lambda_{5}=u_{x}$; two Alfvén waves traveling with speed $\lambda_{3,7}=u \mp c_{a}$; and four magneto-acoustic waves, two fast and two slow with speeds $\lambda_{2,8}=u \mp c_{f}$ and $\lambda_{4,6}=u \mp c_{s}$, respectively, where

$$
c_{a}=\frac{\left|B_{x}\right|}{\sqrt{\rho}}, \quad c_{f, s}^{2}=\frac{1}{2}\left(\frac{\gamma p+\mathbf{B} \cdot \mathbf{B}}{\rho} \pm \sqrt{\left(\frac{\gamma p+\mathbf{B} \cdot \mathbf{B}}{\rho}\right)^{2}-4 \frac{\gamma p B_{x}^{2}}{\rho^{2}}}\right) .
$$

From the decoupled system, we obtain the eigenvalues $\lambda_{1,9}=\mp c_{h}$, which are distinct from the eigenvalues of $\mathcal{A}^{\prime}$ for a sufficiently large $c_{h}$. Therefore, the matrix $\mathcal{A}$ has nine eigenvalues, such that

$$
\lambda_{1} \leq \lambda_{2} \leq \lambda_{3} \leq \lambda_{4} \leq \lambda_{5} \leq \lambda_{6} \leq \lambda_{7} \leq \lambda_{8} \leq \lambda_{9}
$$

We draw analogous results for matrices $\mathcal{B}$ and $\mathcal{C}$. Therefore, we find that system (20) is hyperbolic. 


\subsection{Numerical approximation}

In the previous section, we obtained the eigenvalues $\lambda_{1,9}=\mp c_{h}$ from the decoupled system, where the constant $c_{h}$ represents the propagation speed of local divergence errors. Thus, $c_{h}$ is chosen to be the maximum signal speed compatible with the time step $\Delta t$, such that

$$
c_{h}=\max _{i, j, k}\left(\left|u_{x}\right|+c_{f, x},\left|u_{y}\right|+c_{f, y},\left|u_{z}\right|+c_{f, z}\right),
$$

where $c_{f, x}, c_{f, y}$ and $c_{f, z}$ are the fast magneto-acoustic speeds in the three directions. The time increment is restricted by the Courant-Friedrichs-Levy $(\mathrm{CFL})$ condition $c_{c f l} \in(0,1)$ in the following way:

$$
\Delta t=c_{c f l} \frac{\min (\Delta x, \Delta y, \Delta z)}{c_{h}} .
$$

We attempt to solve equation (21), using the Godunov-type approach explained in Section 2. Hence, it is necessary to find a numerical flux for the GLM-MHD system and we begin by deriving it for the hyperbolic GLM-MHD system, i.e., system (20) with no source terms. First, we notice that for arbitrary left and right states $\left(B_{x, L}, \psi_{L}\right)$ and $\left(B_{x, R}, \psi_{R}\right)$, the Godunov flux of system (24) can be computed exactly since

$$
\left(\begin{array}{c}
\tilde{B}_{x} \\
\tilde{\psi}
\end{array}\right)=\left(\begin{array}{c}
\frac{1}{2}\left(B_{x, L}+B_{x, R}\right)-\frac{1}{2 c_{h}}\left(\psi_{R}-\psi_{L}\right) \\
\frac{1}{2}\left(\psi_{L}+\psi_{R}\right)-\frac{c_{h}}{2}\left(B_{x, R}-B_{x, L}\right)
\end{array}\right) .
$$

Therefore we derive the numerical flux $\left(\tilde{\psi}, c_{h}^{2} \tilde{B}_{x}\right)^{T}$. For the remaining system, we use a seven-wave Riemann solver $\mathcal{R}$ for the one-dimensional MHD equations with the normal component of the magnetic field defined by $\tilde{B_{x}}$. Hence, the numerical flux $\tilde{\mathcal{G}}_{x}$ has the following form:

$$
\tilde{\mathcal{G}}_{x}=\mathcal{G}_{x}\left(\mathcal{R}\left(0 ; \mathbf{V}_{L}, \mathbf{V}_{R}, \tilde{B}_{x}\right), 0\right)+\left(0,0,0,0, \tilde{\psi}, 0,0,0, c_{h}^{2} \tilde{B}_{x}\right)^{T},
$$

and, analogous expressions can be found for $\tilde{\mathcal{G}}_{y}$ and $\tilde{\mathcal{G}}_{z}$. Moreover, for the mixed GLM-MHD system, which considers the source terms in the right-hand side of system (20), we use an operator-splitting approach. Thus, in the source step, we solve the initial value problem

$$
\partial_{t} \psi=-\frac{c_{h}^{2}}{c_{p}^{2}} \psi
$$

for which the initial condition $\psi^{*}$ is the output of the previous step. Integrating exactly for a time increment $\Delta t$, yields $\psi^{n+1}=\psi^{*} \exp \left(-\Delta t c_{h}^{2} / c_{p}^{2}\right)$. Dedner et al. recommend fixing the value $c_{p}^{2} / c_{h}=0.18$.

\subsection{Boundary conditions}

For the magnetohydrodynamic variables considered in system (1)-(4), the initial and boundary conditions are chosen according to the specific physical settings of the problem under consideration, but for the variable $\psi$, we are free to prescribe them. Given its nature, a good choice for the initial value of the unphysical variable is $\psi^{0}=0$. Regarding the particular choice of the boundary condition, Dedner et al. recommend assuming that the behavior of $\psi$ and $\rho$ is identical at the boundary, making the implementation quite simple and straightforward on an existing code.

\section{Constrained transport}

The constrained transport (CT) method for ideal MHD, devised by Evans and Hawley [6], is a numerical scheme that applies a staggered mesh (see Section 4.1) to evolve the induction equation while maintaining 
the divergence-free property of the magnetic field to machine round-off error precision. We use the notation introduced in Section 2. In order to avoid confusion, from now on, we denote the cell-centered magnetic field by the usual capital $\mathbf{B}$ and the staggered magnetic field representation by a lowercase $\mathbf{b}$.

\subsection{Staggered mesh discretization}

The staggered mesh formulation simply consists in defining the magnetic field components at the cell interfaces, the electric fields at the zone edges, and all the hydrodynamic variables at the centers. Figure 4.1 shows the collocation of the magnetic and electric field in two dimensions, with the magnetic field components $b_{x}$ and $b_{y}$ defined on the interface centers to which they are orthogonal. The $z$ component of the electric field is located at the cell corners.

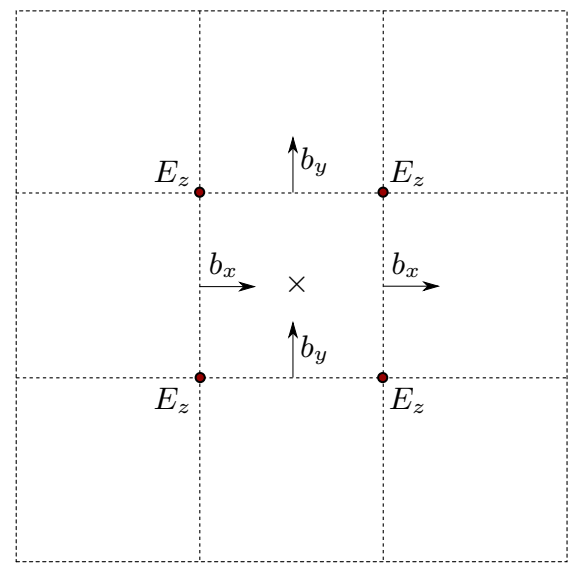

FIgURE 4.1. Two-dimensional staggering in the constrained transport approach.

The main justification for using a staggered algorithm is that it allows to define an inherently divergencefree method. Recalling Faraday's equation (6), it is clear that a discrete version of Stokes' theorem may be used to evolve in time a magnetic field that has a staggered representation. Let us consider a cell $(i, j)$ in two dimensions, with the staggered magnetic field components defined by $b_{x, i-1 / 2, j}, b_{x, i+1 / 2, j}, b_{y, i, j-1 / 2}$ and $b_{y, i, j+1 / 2}$. As mentioned above, the electric field components are placed at cell corners . Hence, the induction equation is discretized along the cell edges as

$$
\begin{aligned}
& b_{x, i-1 / 2, j}^{n+1}=b_{x, i-1 / 2, j}^{n}-\frac{\Delta t}{\Delta y}\left[E_{z, i-1 / 2, j+1 / 2}-E_{z, i-1 / 2, j-1 / 2}\right], \\
& b_{x, i+1 / 2, j}^{n+1}=b_{x, i+1 / 2, j}^{n}-\frac{\Delta t}{\Delta y}\left[E_{z, i+1 / 2, j+1 / 2}-E_{z, i+1 / 2, j-1 / 2}\right], \\
& b_{y, i, j-1 / 2}^{n+1}=b_{y, i, j-1 / 2}^{n}+\frac{\Delta t}{\Delta x}\left[E_{z, i+1 / 2, j-1 / 2}-E_{z, i-1 / 2, j-1 / 2}\right], \\
& b_{y, i, j+1 / 2}^{n+1}=b_{y, i, j+1 / 2}^{n}+\frac{\Delta t}{\Delta x}\left[E_{z, i+1 / 2, j+1 / 2}-E_{z, i-1 / 2, j+1 / 2}\right] .
\end{aligned}
$$

If we define the numerical divergence $\nabla \cdot \mathbf{b}$ for cell $(i, j)$ at time $t^{n}$ as

$$
(\nabla \cdot \mathbf{b})_{i, j}^{n}=\frac{b_{x, i+1 / 2, j}^{n}-b_{x, i-1 / 2, j}^{n}}{\Delta x}+\frac{b_{y, i, j+1 / 2}^{n}-b_{y, i, j-1 / 2}^{n}}{\Delta y}
$$

it is quite easy to show that an initial $(\nabla \cdot \mathbf{b})_{i, j}^{n}=0$ leads to $(\nabla \cdot \mathbf{b})_{i, j}^{n+1}=0$, with machine round-off error accuracy, i.e., the staggered approach maintains the constraint equation to machine round-off. 


\subsection{Numerical approximation}

We briefly describe a finite volume time-update strategy, with the purpose of showing the main steps needed to evolve the state variables over one time step. At the beginning of the time step, the hydrodynamic variables are defined at the center of the cells and the magnetic field $\mathbf{b}$ at the corresponding interface centers. The initial magnetic field at the cell center $\mathbf{B}_{i, j, k}$ may be approximately obtained in the following way:

$$
B_{x, i, j, k}^{n}=\frac{b_{x, i-1 / 2, j, k}^{n}+b_{x, i+1 / 2, j, k}^{n}}{2}, B_{y, i, j, k}^{n}=\frac{b_{y, i, j-1 / 2, k}^{n}+b_{y, i, j+1 / 2, k}^{n}}{2}, B_{z, i, j, k}^{n}=\frac{b_{z, i, j, k-1 / 2}^{n}+b_{z, i, j, k+1 / 2}^{n}}{2} .
$$

Let us denote the vector of centered variables by $\mathbf{U}_{i, j, k}^{n}=\left(\rho_{i, j, k}, \rho_{i, j, k} \mathbf{u}_{i, j, k}, \varepsilon_{i, j, k}, \mathbf{B}_{i, j, k}\right)^{T}$. We then find the fluxes at the faces by means of a seven-wave Riemann solver, as in (9), and make the update of the state vector $\mathbf{U}_{i, j, k}^{n}$ using expression (10) in order to obtain $\mathbf{U}_{i, j, k}^{n+1}$. At this point, what remains is to update the magnetic field components at the faces. The main idea consists on constructing an approximation to the electric field defined in (7) at the cell corners. This approximated electric field is then used to update the face centered magnetic fields, by using a discrete version of Stokes' theorem, as shown in example (27).

A simple approach for the estimation of the electromotive force (EMF) at the cell edges is based on a simple spatial interpolation at time $t^{n}$. In the following example, we approximate the EMF $E_{z, i-1 / 2, j-1 / 2}^{n}$ that is needed in (27). Thus,

with

$$
E_{z, i-1 / 2, j-1 / 2}^{n}=\bar{u}_{x, i-1 / 2, j-1 / 2} \bar{B}_{y, i-1 / 2, j-1 / 2}-\bar{u}_{y, i-1 / 2, j-1 / 2} \bar{B}_{x, i-1 / 2, j-1 / 2}
$$

$$
\begin{gathered}
\bar{u}_{x, i-1 / 2, j-1 / 2}=\frac{u_{x, i, j, k}^{n}+u_{x, i-1, j, k}^{n}+u_{x, i, j-1, k}^{n}+u_{x, i-1, j-1, k}^{n}}{4}, \quad \bar{B}_{x, i-1 / 2, j-1 / 2}=\frac{B_{x, i-1 / 2, j, k}^{n}+B_{x, i-1 / 2, j-1, k}^{n}}{2}, \\
\bar{u}_{y, i-1 / 2, j-1 / 2}=\frac{u_{y, i, j, k}^{n}+u_{y, i-1, j, k}^{n}+u_{y, i, j-1, k}^{n}+u_{y, i-1, j-1, k}^{n}}{4}, \quad \bar{B}_{y, i-1 / 2, j-1 / 2}=\frac{B_{y, i, j-1 / 2, k}^{n}+B_{y, i-1, j-1 / 2, k}^{n}}{2} .
\end{gathered}
$$

It is important to mention that several and more complex methods have been proposed to update the magnetic fields at the interface centers. We refer the reader to $[1,6,7,9,14]$.

\section{NumericAl RESULTS}

The numerical implementation of both methods presented in this paper has been done in the HERACLES code for astrophysical fluid flows. By having a common computational framework, we can fairly compare the accuracy and robustness of the hyperbolic divergence cleaning and constrained transport techniques. Thus, in this section, we present a series of selected test problems. We note that the divergence of the magnetic field $\mathbf{B}$ for cell $(i, j, k)$ at time $t^{n}$ is computed as

$$
(\nabla \cdot \mathbf{B})_{i, j, k}^{n}=\frac{B_{x, i+1, j, k}^{n}-B_{x, i-1, j, k}^{n}}{2 \Delta x}+\frac{B_{y, i, j+1, k}^{n}-B_{y, i, j-1, k}^{n}}{2 \Delta y}+\frac{B_{z, i, j, k+1}^{n}-B_{z, i, j, k-1}^{n}}{2 \Delta z} .
$$

For second order approximations, we extend the hyperbolic cleaning scheme by using the MUSCL-Hancock Method (MHM), see [13]. In the case of the constrained transport, the MUSCL-Hancock approach detailed in [7] is employed. As for the choice of slope limiters, we use the MinMod limiter since it is known to ensure the positivity of the solution in multiple space dimensions.

\subsection{Advection in $B_{x}$}

This problem, summarized in Table 1, has a non-zero initial divergence of the magnetic field. Thus, the purpose of performing tests for this unphysical problem is to determine whether the divergence cleaning technique is robust enough or not and we show results obtained using the first order hyperbolic and mixed GLM approaches. 
In the contour plots shown in Figure 5.1, we can perceive that during the time evolution, the initial peak in $B_{x}$ decreases in height for both the hyperbolic and mixed cleaning, but is well advected with the flow velocity nonetheless. The mixed GLM solutions do not show the complex wave interactions seen in the hyperbolic case, because of the additional damping. Additionally, this problem also allows to find the optimal value for the ratio $c_{p}^{2} / c_{h}=0.18$ (see Figure 5.2).

\section{Advection in $B_{x}$}

Computational domain: $[-0.5,1.5] \times[-0.5,1.5]$; Periodic boundaries

\begin{tabular}{cccccccc}
$\rho$ & $u_{x}$ & $u_{y}$ & $u_{z}$ & $B_{x}$ & $B_{y}$ & $B_{z}$ & $p$ \\
\hline 1.0 & 1.0 & 1.0 & 0.0 & $r\left(x^{2}+y^{2}\right) / \sqrt{4 \pi}$ & 0.0 & $1 / \sqrt{4 \pi}$ & 6.0 \\
$r(s)=\left\{\begin{array}{lll}4096 s^{4}-128 s^{2}+1 & \text { if } s \in[0,0.125], \\
0 & & \text { otherwise. }\end{array}\right.$
\end{tabular}

TABLE 1. Initial data for the peak in $B_{x}$ problem described in [5].
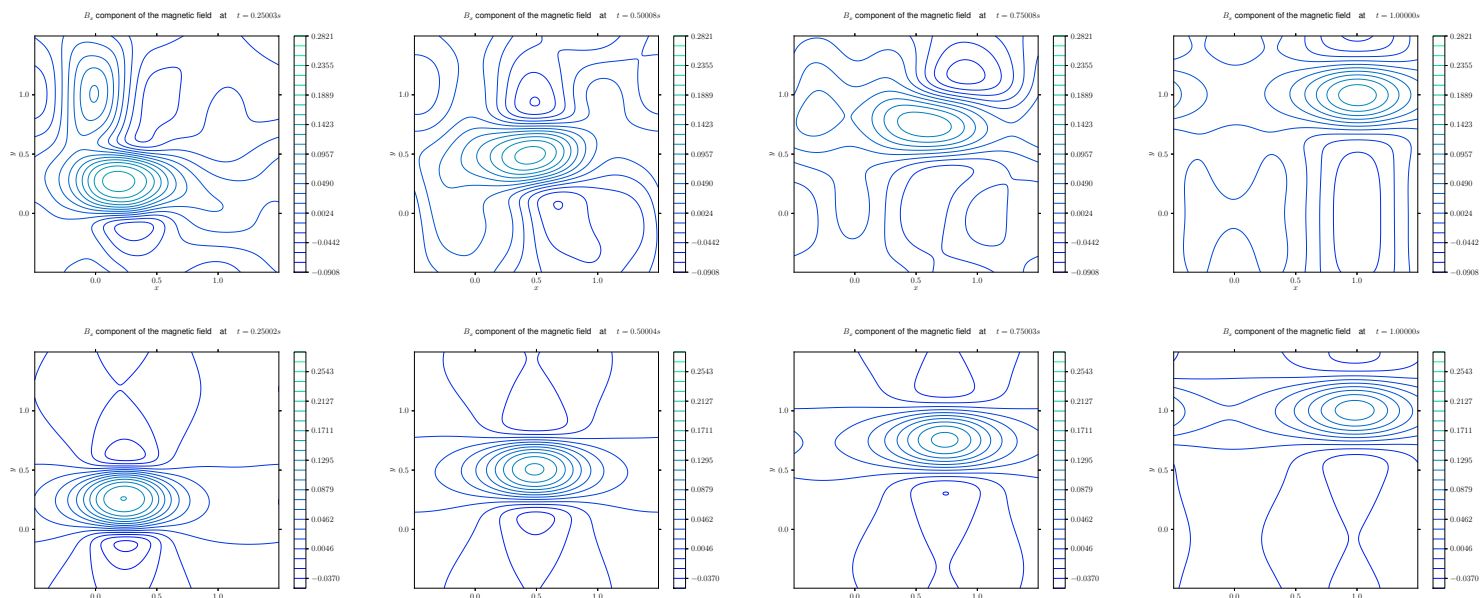

(a) $t=0.25$

(b) $t=0.50$

(c) $t=0.75$

(d) $t=1.0$

FIgURE 5.1. Isolines of $B_{x}$ obtained with the HLLD scheme. The computations are performed with $256 \times 256$ points for hyperbolic and mixed GLM approaches (from top to bottom).

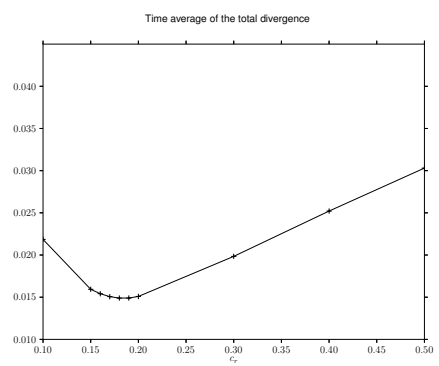

(a) $64 \times 64$ points

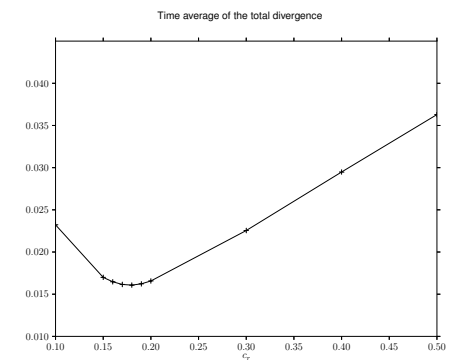

(b) $128 \times 128$ points

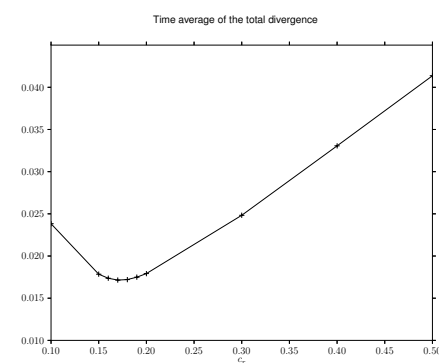

(c) $256 \times 256$ points

FIGURE 5.2. Time averages of the total divergence obtained with the HLLD scheme for problem 5.1 using different values of $c_{r}=c_{p}^{2} / c_{h}$. The optimal value is about 0.18 , independent of the grid resolution. 


\subsection{Orszag-Tang}

The Orszag-Tang vortex is a standard and well-known 2D test for MHD codes. It describes a periodic fluid configuration with initial conditions that lead to a system of supersonic MHD turbulence (see Table 5.2). Thus, this problem allows to test the different methods' ability to handle such turbulence and MHD shocks.

\begin{tabular}{lccccccc}
\multicolumn{8}{c}{ Orszag-Tang } \\
\hline \multicolumn{7}{c}{ Computational domain: } & {$[0,1] \times[0,1] ;$ Periodic boundaries } \\
\hline$\rho$ & $u_{x}$ & $u_{y}$ & $u_{z}$ & $B_{x}$ & $B_{y}$ & $B_{z}$ & $p$ \\
\hline$\gamma^{2}$ & $-\sin (2 \pi y)$ & $\sin (2 \pi x)$ & 0.0 & $-\sin (2 \pi y)$ & $\sin (4 \pi x)$ & 0.0 & $\gamma$ \\
\hline TABLE 2. Initial data for the Orszag-Tang vortex described in $[10]$
\end{tabular}

Density distributions at times $t=0.5$ and $t=1.0$ are shown in Figure 5.3, where we can visualize the formation of small scale vortices and turbulence. The good agreement between our results and the ones obtained in previous investigations, such as in $[4,7,10,11,14]$, is satisfactory.

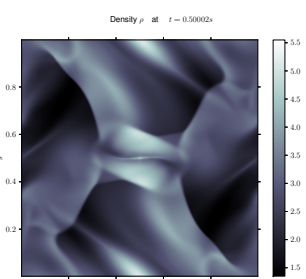

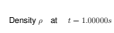

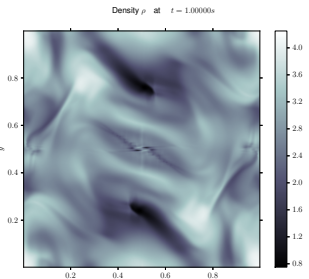

(a) No correction

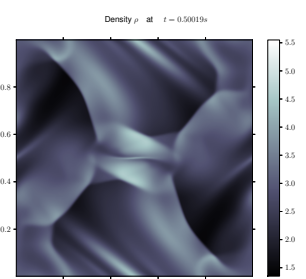

Desisty at $t-1$. 1.momos

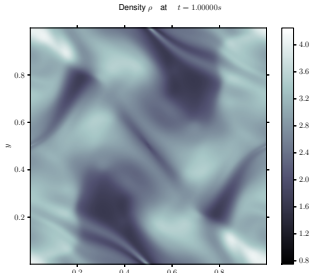

(b) Hyperbolic GLM
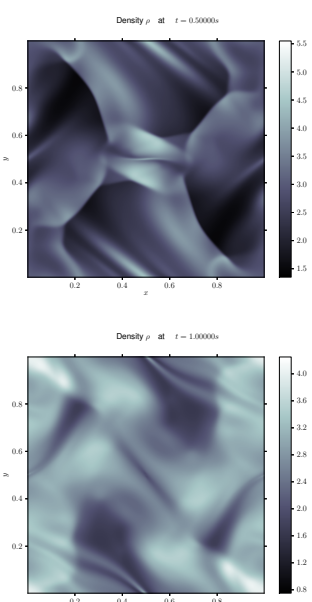

(c) Mixed GLM
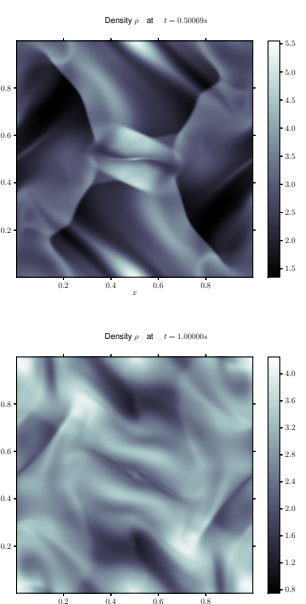

(d) $\mathrm{CT}$

FIGURE 5.3. 2D density plots, first order in both space and time, for the Orszag-Tang system using $256 \times 256$ points at times $t=0.5$ (top) and $t=1.0$ (bottom).

In Figure 5.4, the evolution of the $L^{1}$ norm and maximum value of the divergence is plotted for different cell-centered techniques. It is evident that the measured $L^{1}$ errors for the hyperbolic and mixed approaches seem to converge to zero as time increases, while those obtained without correction tend to increase with time. We note that a second order simulation with no correction is not possible to obtain since the blow-up of divergence errors causes the crash of the simulation. In Figure 5.5, we show horizontal cuts at $y=0.3125$ of the pressure distribution, and find no perceivable difference between the hyperbolic and mixed GLM techniques. Moreover, the same figure allows to conclude that the constrained transport method solves this problem more accurately than the divergence cleaning techniques presented in this paper.

\subsection{Kelvin-Helmholtz Instability}

As a final test, we simulate the MHD Kelvin-Helmholtz instability in two dimensions. This instability has been widely studied in the literature and it clearly shows the advantages of maintaining a divergence-free magnetic field. The initial conditions are given in Table 5.3, with the inital perturbation depicted in Figure 5.6. 

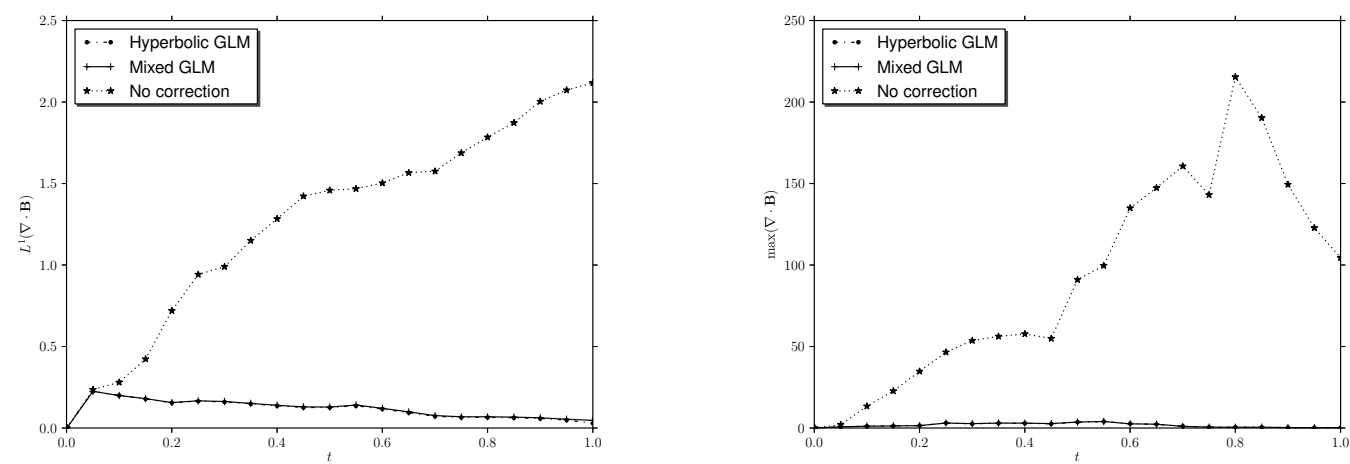

FIGURE 5.4. $L^{1}(\nabla \cdot \mathbf{B})$ (left) and $\max (\nabla \cdot \mathbf{B})$ (right) obtained with the HLLD scheme for the OrszagTang vortex. The computations are performed using a cell-centered approach on $256 \times 256$ points.

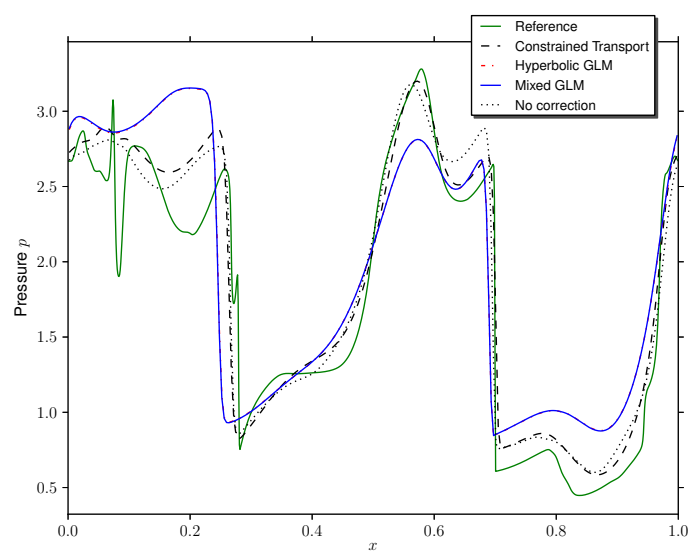

(a) First order

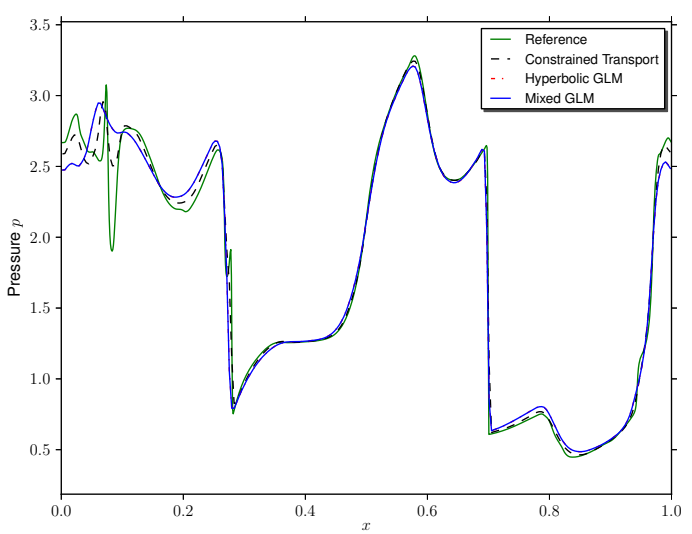

(b) Second order

FigURE 5.5. Horizontal cut at $y=0.3125$ showing the gas pressure $p$ in the Orszag-Tang system at $t=0.5$ using the HLLD scheme and $256 \times 256$ points. The solid green line gives a reference solution obtained with a second-order constrained transport algorithm on a finer grid of $1024 \times 1024$ points.

\section{Kelvin-Helmholtz Instability}

$$
\text { Computational domain: }[0,1] \times[-1,1]
$$

Boundaries: reflexive on top and bottom, periodic on left and right

\begin{tabular}{rccccccc}
\hline$\rho$ & $u_{x}$ & $u_{y}$ & $u_{z}$ & $B_{x}$ & $B_{y}$ & $B_{z}$ & $p$ \\
\hline 1.0 & $M / 2(\tanh (20 y))$ & 0.0 & 0.0 & $c_{a} \sqrt{\rho} \cos (\theta)$ & 0.0 & $c_{a} \sqrt{\rho} \sin (\theta)$ & $1 / \gamma$ \\
$\theta=\pi / 3$, & $M=1$, & $c_{a}=0.1$, & $\sigma=0.1$ \\
\hline
\end{tabular}

Single mode perturbation: $u_{y}(x, y)=0.1 \sin (2 \pi x)\left(e^{-y^{2} / \sigma^{2}}\right)$

TABLE 3. Initial data for the Kelvin-Helmholtz instability described in [10]. 

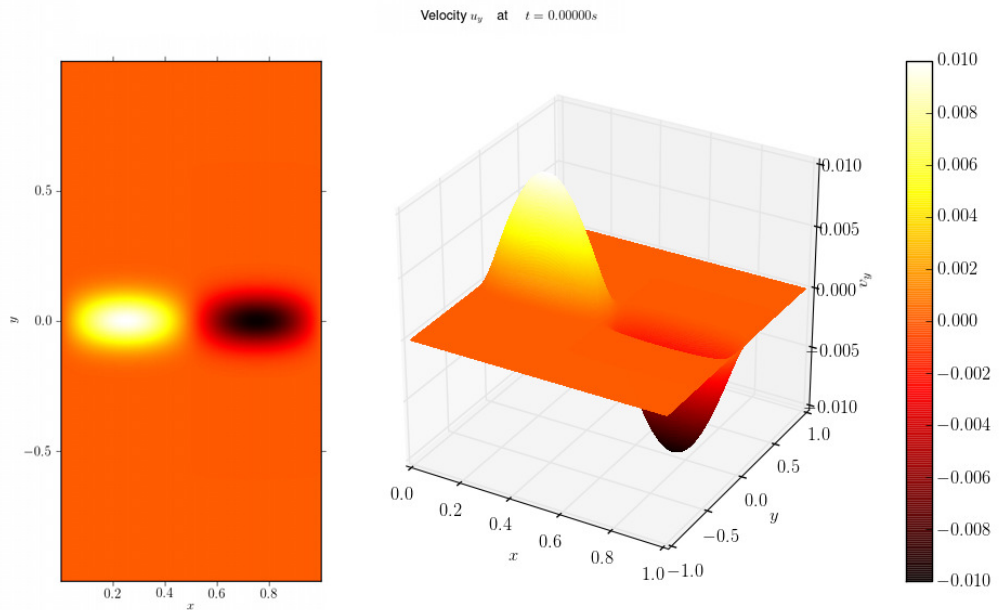

FIGURE 5.6. Initial single mode perturbation $u_{y}(x, y)$ for the Kelvin-Helmholtz instability.

The left plot in Figure 5.7 shows the $L^{1}$ norm of the divergence $\nabla \cdot \mathbf{B}$ at different times for the methods that use a cell-centered collocation. For the case without correction, a blow-up of divergence errors occurs, causing the simulation to crash. This problem is then addressed by adding a divergence cleaning technique. Additionally, on the right plot, we present the time evolution of the $L^{1}$ norm of the total energy density $\varepsilon$, a conserved quantity in the MHD equations. However, for the constrained transport method, there is a slight loss of the conservation at the level of discretization error.
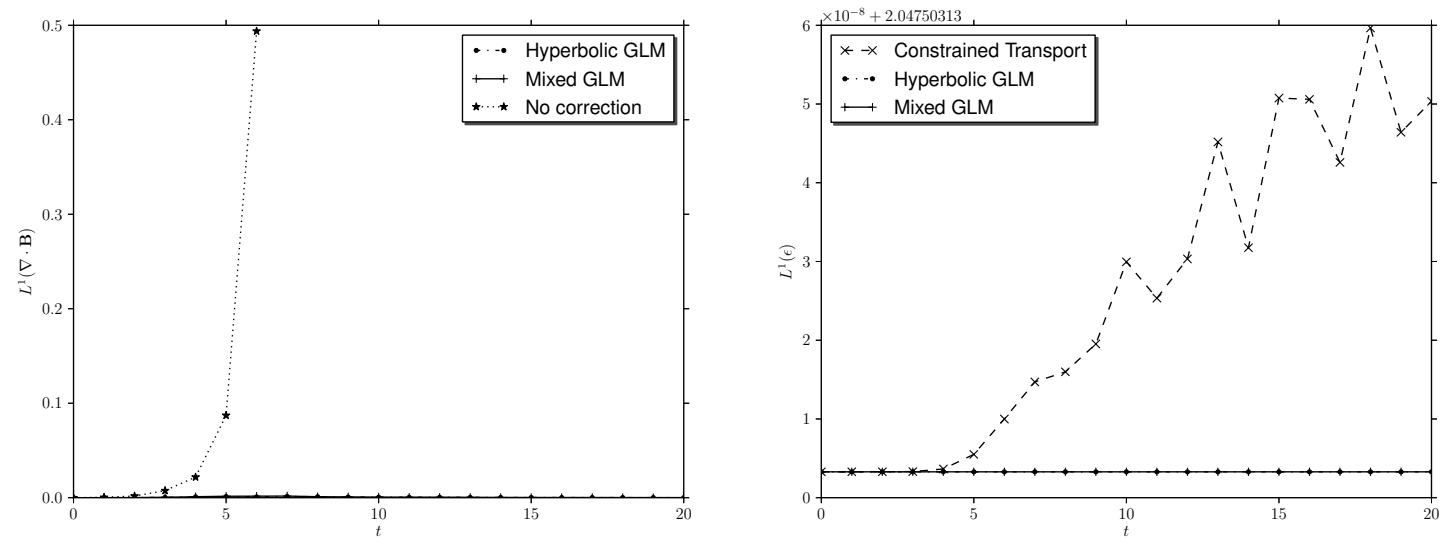

FiguRE 5.7. $L^{1}(\nabla \cdot \mathbf{B})$ (left) and $L^{1}(\varepsilon)$ (right) obtained with the HLLD scheme for the KelvinHelmholtz instability. The computations are performed using $256 \times 256$ points.

Evolution plots are shown in Figure 5.8 for the ratio of the poloidal field strength and the toroidal component $\sqrt{B_{x}^{2}+B_{y}^{2}} / B_{z}$. For all the methods analyzed, in the first five seconds we perceive the formation of the typical cat's eye vortex structure, as expected. We note that, for both first and second order accuracy, the hyperbolic divergence cleaning technique preserves the conservation of the total energy density. However, it tends to be more diffusive than the constrained transport method. 

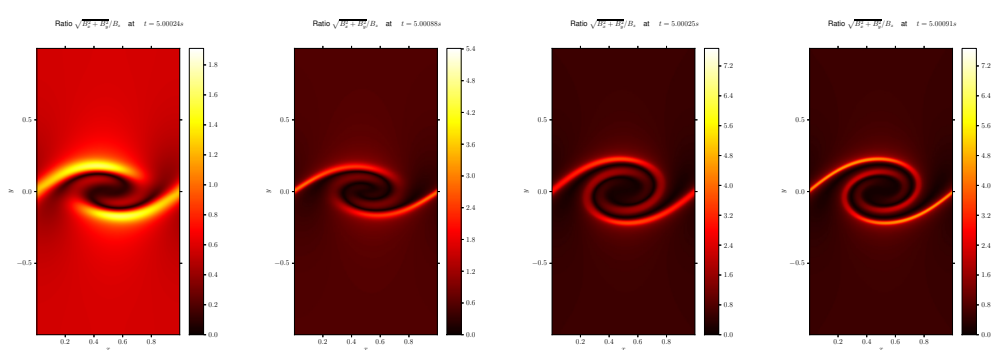

(a) $t=5$
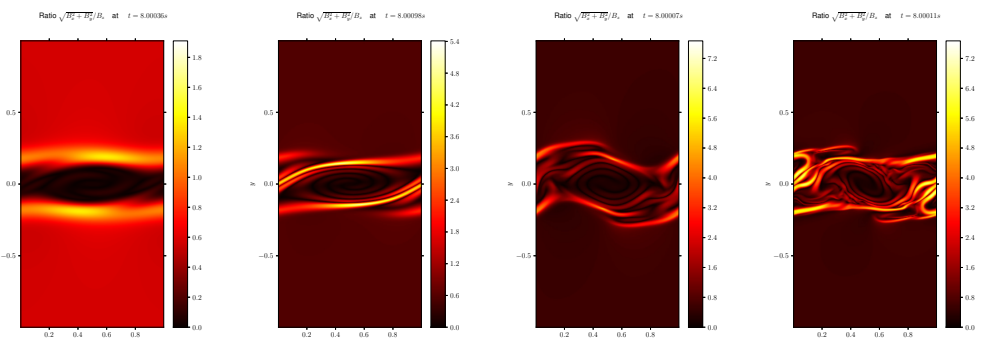

(b) $t=8$
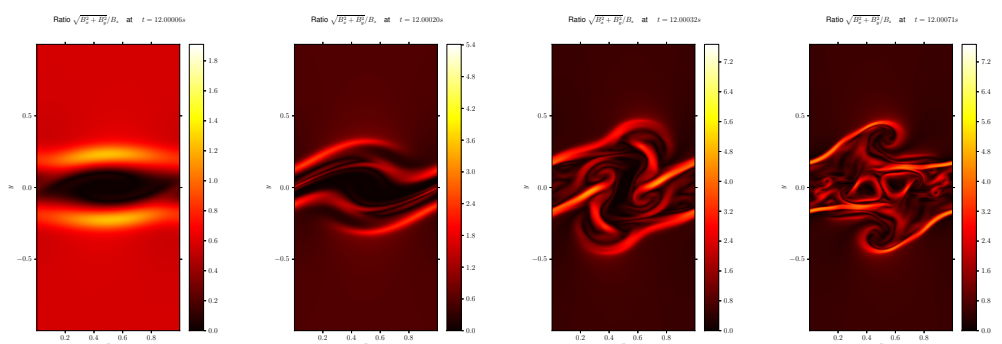

(c) $t=12$
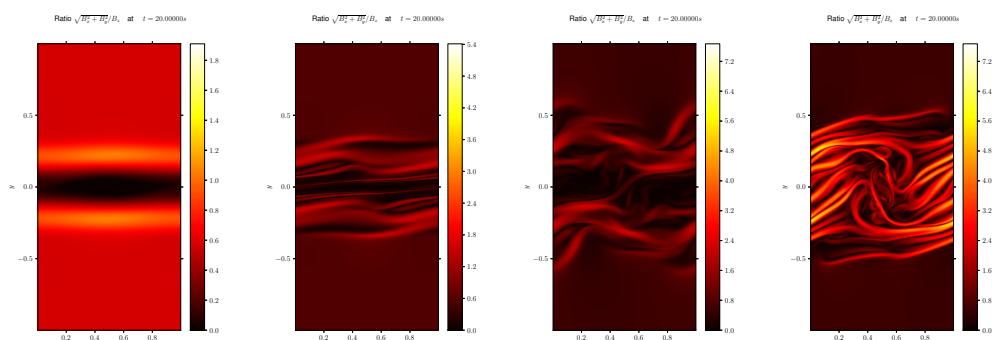

(d) $t=20$

FIGURE 5.8. Evolution of the Kelvin-Helmholtz instability obtained with the HLLD scheme for the mixed GLM, constrained-transport, second order mixed GLM, and second order constrained-transport (from left to right). The results for the hyperbolic GLM (not shown) are almost identical to those obtained with the mixed GLM technique. The plots show the ratio of the poloidal field strength and the toroidal component, i.e., $\sqrt{B_{x}^{2}+B_{y}^{2}} / B_{z}$. The computations are done on a mesh of $256 \times 512$ points. 


\section{Conclusions}

In this paper, we have investigated and compared two different methods that aim to maintain the divergencefree property of the magnetic field, a constraint that cannot be ignored without having consequences.

The method proposed by Dedner et al. [5] prescribes a hyperbolic equation that allows for the divergence errors to be propagated to the boundary of the domain. The same authors recommend using a small variation of this approach, the mixed GLM ansatz, which offers both propagation and damping of the errors. The advantage of the divergence cleaning technique is that it is easy to implement as it is based on the cell-centered formulation favored in the Godunov approach. However, one disadvantage is that it depends on tunable parameters.

On the other hand, the constrained transport (CT) approach, originally introduced by Evans and Hawley [6], relies on a staggered formulation of the magnetic and electric fields. One clear advantage of this method is its inherently divergence-free magnetic field. Moreover, it does not have tunable parameters, as in the hyperbolic divergence cleaning technique. However, this method is harder to implement and it sometimes presents loss of the conservation of the total energy density.

Through the different numerical test cases, we have shown that the implementation of the hyperbolic divergence cleaning approach in the HERACLES code was successful. This has allowed us to compare both methods and comment on the advantages and disadvantages that they possess. We were able to reproduce quantitatively results obtained by several authors and found that both methods are robust and efficient. Although we find that the hyperbolic divergence cleaning generates more diffusive results than the constrained transport method, the simplicity of the method makes it an attractive technique for our future work in the design of a high order finite volume approximation for hyperbolic conservation laws in curvilinear unstructured grids.

\section{REFERENCES}

[1] D. S. Balsara and D. S. Spicer. A staggered mesh algorithm using high order Godunov fluxes to ensure solenoidal magnetic fields in magnetohydrodynamic simulations. Journal of Computational Physics, 149(2):270-292, 1999.

[2] T. Barth. On the role of involutions in the discontinuous Galerkin discretization of Maxwell and magnetohydrodynamic systems. In Compatible Spatial Discretizations, volume 142 of The IMA Volumes in Mathematics and its Applications, pages 69-88. Springer New York, 2006.

[3] J. U. Brackbill and D. C. Barnes. The effect of nonzero $\nabla \cdot \mathbf{B}$ on the numerical solution of the magnetohydrodynamic equations. Journal of Computational Physics, 35(3):426-430, 1980.

[4] W. Dai and P. R. Woodward. On the divergence-free condition and conservation laws in numerical simulations for supersonic magnetohydrodynamical flows. The Astrophysical Journal, 494(1):317, 1998.

[5] A. Dedner, F. Kemm, D. Kröner, C.-D. Munz, T. Schnitzer, and M. Wesenberg. Hyperbolic divergence cleaning for the MHD equations. Journal of Computational Physics, 175(2):645-673, 2002.

[6] C. R. Evans and J. F. Hawley. Simulation of magnetohydrodynamic flows: a constrained transport method. Astrophysical Journal, Part 1, 332:659-677, September 1988.

[7] S. Fromang, P. Hennebelle, and R. Teyssier. A high order Godunov scheme with constrained transport and adaptive mesh refinement for astrophysical MHD. A\& $A$, 457:371-384, 2006.

[8] C. Helzel, J. A. Rossmanith, and B. Taetz. An unstaggered constrained transport method for the 3D ideal magnetohydrodynamic equations. Journal of Computational Physics, 230(10):3803-3829, 2011.

[9] P. Londrillo and L. Del Zanna. High-order upwind schemes for multidimensional magnetohydrodynamics. The Astrophysical Journal, $530(1): 508,2000$.

[10] A. Mignone, P. Tzeferacos, and G. Bodo. High-order conservative finite difference GLM-MHD schemes for cell-centered MHD. Journal of Computational Physics, 229(17):5896-5920, 2010.

[11] T. Miyoshi and K. Kusano. A multi-state HLL approximate Riemann solver for ideal magnetohydrodynamics. Journal of Computational Physics, 208(1):315-344, 2005.

[12] K. G. Powell. An approximate Riemann solver for magnetohydrodynamics (that works in more than one dimension). Technical Report 94-24, ICASE, Langley, VA, 1994.

[13] E. F. Toro. Riemann Solvers and Numerical Methods for Fluid Dynamics: A Practical Introduction. Springer, 2009.

[14] G. Tóth. The $\nabla \cdot \mathbf{B}=0$ constraint in shock-capturing magnetohydrodynamics codes. Journal of Computational Physics, 161(2):605-652, 2000.

[15] K. S. Yee. Numerical solution of initial boundary value problems involving Maxwell's equations in isotropic media. IEEE Trans. Antennas and Propagation, pages 302-307, 1966. 